\title{
Third Ventricle Pilocytic Astrocytoma
}

National Cancer Institute

\section{Source}

National Cancer Institute. Third Ventricle Pilocytic Astrocytoma. NCI Thesaurus. Code C156037.

A pilocytic astrocytoma occurring in the third ventricle. 